\title{
REVIEW
}

\section{REVISED General guidelines for biomedical software}

\section{development [version 2; peer review: 2 approved]}

\author{
Luis Bastiao Silva(iD1, Rafael C. Jimenez (iD)2, Niklas Blomberg², José Luis Oliveira (iD) 3 \\ ${ }^{1}$ BMD Software, Aveiro, Portugal \\ ${ }^{2}$ ELIXIR Hub, Wellcome Trust Genome Campus, Hinxton, UK \\ ${ }^{3}$ Institute of Electronics and Informatics Engineering of Aveiro, University of Aveiro, Aveiro, Portugal
}

V2 First published: 15 Mar 2017, 6:273
https://doi.org/10.12688/f1000research.10750.1

Latest published: $12 \mathrm{Jul} 2017,6: 273$

https://doi.org/10.12688/f1000research.10750.2

\section{Abstract}

Most bioinformatics tools available today were not written by professional software developers, but by people that wanted to solve their own problems, using computational solutions and spending the minimum time and effort possible, since these were just the means to an end. Consequently, a vast number of software applications are currently available, hindering the task of identifying the utility and quality of each. At the same time, this situation has hindered regular adoption of these tools in clinical practice. Typically, they are not sufficiently developed to be used by most clinical researchers and practitioners. To address these issues, it is necessary to re-think how biomedical applications are built and adopt new strategies that ensure quality, efficiency, robustness, correctness and reusability of software components. We also need to engage end-users during the development process to ensure that applications fit their needs. In this review, we present a set of guidelines to support biomedical software development, with an explanation of how they can be implemented and what kind of open-source tools can be used for each specific topic.

\section{Keywords}

biomedical software, guidelines, software development, bioinformatics, Agile

\author{
Open Peer Review \\ Approval Status \\ 1 \\ 2 \\ version 2 \\ (revision) \\ 12 Jul 2017 \\ version 1 \\ 15 Mar 2017

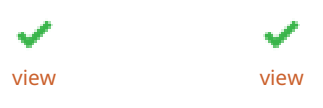 \\ 1. João P. G. L. M. Rodrigues (ID), Stanford \\ University, Stanford, USA \\ 2. Victor Maojo, Universidad Politecnica de \\ Madrid, Madrid, Spain \\ Any reports and responses or comments on the \\ article can be found at the end of the article.
}

This article is included in the Bioinformatics

gateway. 
This article is included in the Bioinformatics

Education and Training Collection collection.

Corresponding authors: Luis Bastiao Silva (bastiao@bmd-software.com), José Luis Oliveira (jlo@ua.pt)

Author roles: Silva LB: Conceptualization, Writing - Original Draft Preparation, Writing - Review \& Editing; Jimenez RC:

Conceptualization, Writing - Original Draft Preparation, Writing - Review \& Editing; Blomberg N: Conceptualization, Writing - Original

Draft Preparation, Writing - Review \& Editing; Luis Oliveira J: Conceptualization, Writing - Original Draft Preparation, Writing - Review \& Editing

Competing interests: No competing interests were disclosed.

Grant information: This work has partially received funding from the European Union's Horizon 2020 Research and Innovation programme for 2014-2020 under Grant Agreement n. 634143 (MedBioinformatics) and from the EU/EFPIA Innovative Medicines Initiative Joint Undertaking (EMIF grant $\mathrm{n}^{\circ} 115372$ ).

The funders had no role in study design, data collection and analysis, decision to publish, or preparation of the manuscript.

Copyright: @ 2017 Silva LB et al. This is an open access article distributed under the terms of the Creative Commons Attribution License, which permits unrestricted use, distribution, and reproduction in any medium, provided the original work is properly cited.

How to cite this article: Silva LB, Jimenez RC, Blomberg N and Luis Oliveira J. General guidelines for biomedical software

development [version 2; peer review: 2 approved] F1000Research 2017, 6:273 https://doi.org/10.12688/f1000research.10750.2

First published: 15 Mar 2017, 6:273 https://doi.org/10.12688/f1000research.10750.1 


\section{REVISED Amendments from Version 1}

The authors are grateful for the suggestions of the two referees, which we have addressed in this revision.

A better clarification of the terms bioinformatics and biomedical informatics, is now provided. We have also compared the differences, regarding software development, between the academia and software companies.

Figure 1 was redrawn, according to the referee suggestion, with examples of tools that can be used in the different development phases.

The recommendation about source code management was also clarified.

Overall, other improvements and minor corrections were also addressed.

We also would like to thank to Peter Rijnbeek for provide us great feedback that allow us to improve the manuscript.

See referee reports

\section{Introduction}

As an increasing number of scientific results are being generated from omics studies, new translational medicine applications and bioinformatics tools are needed to promote the flow of these results into clinical practice, i.e. the knowledge needs to be translated from the bench to the bedside, to foster development of new biotechnological products and improve patients' health. Biomedical informatics intends to support the integration and transfer of knowledge across all major subject areas of translational medicine - from the study of individual molecules to the study of whole populations ${ }^{1}$. Translational medicine brings together many areas of informatics, including bioinformatics, imaging informatics, clinical informatics and public health informatics ${ }^{2,3}$. Bioinformaticians, translational researchers and computational biologists identify the molecular and cellular components that can be targeted for specific clinical interventions and treatments for specific diseases. Imaging informatics also plays a significant role in understanding pathogenesis and identifying treatments at the molecular, cellular, tissue and organ level. Richer methods to visualize and analyse imaging data are already being investigated and developed ${ }^{4}$. Other techniques such as text and data mining have been applied to clinical reports. Additionally, translational research teams need to focus on decision support, natural language processing (NLP), standards, information retrieval before applying these techniques to the electronic health records.

The biomedical informatics landscape is pushing for the development of more professional and easy-to-use software applications, in order to address the pressing need to translate research outcomes into clinical practice. To accomplish this, solid software engineering approaches must be adopted. Despite being a relatively young discipline, biomedical informatics has evolved at an impressive rate, constantly creating new software solutions and tools. However, due to their multidisciplinary nature, it is often difficult for individual studies to gather solid knowledge in their various fields. This problem has been flagged by several authors, who have proposed general competences that undergraduate students should acquire $^{5,6}$. These competences can be obtained through introducing complementary courses, such as software programming, in existing curricula, or by creating new academic degree courses ${ }^{7}$. While these strategies have resulted in many new and successful graduates, the right balance between looking for strong expertise in a single topic, or medium expertise in many topics, is not always easy to find. Nonetheless, it is important to address that there is a clear difference when software developers work for an academic thesis or project, compared to working in software companies. The academia projects are mostly frequent focused on the scientific novelty, while companies are more focused on achieving concrete results for the market needs. In both scenarios, software development methodologies need to be taken at distinct levels of complexity ${ }^{8}$.

Many researchers without training in software engineering have found themselves faced with the intricate task of building their own software solutions. Moreover, researchers and clinicians typically perceive software development as an auxiliary task to serve science, rather than a central goal ${ }^{9}$. The result is sometimes code-difficult and costly to maintain and re-use. This software dependency is indeed a problem across all science, where concerns about the reproducibility of research have raised the need for robust, open access and open source software ${ }^{10,11}$. The development of software projects requires effective collaboration between users and software developers, and also between the users themselves.

Another common drawback of current bioinformatics and clinical applications is the lack of user-friendly interfaces, making them difficult to use and navigate. User-centered design has also been proposed as a way to minimize this problem ${ }^{12}$. The development of open source solutions has promoted software quality in the field, since it encourages public review, reuse, correction and continuous extension ${ }^{13}$.

In concrete for bioinformatics, most of the software is written by researchers who use it for their own individual purposes, a process long-identified as end-user programming ${ }^{14}$. However, these "new" programmers face many software engineering challenges, such as making decisions about design, reuse, integration, testing, and debugging ${ }^{15}$. Several authors have tried to introduce software engineering approaches in bioinformatics programming to address this problem. Hastings et al. ${ }^{16}$ compiled several recommendations that should be used to ensure the usability and sustainability of research software. Most of these suggestions are part of fundamental programming principles; e.g. keep simple, avoid repetitions, avoid spaghetti code. By examining a group of software projects, Rother et al. also identified a set of techniques that facilitate the introduction of software engineering approaches in academic projects ${ }^{17}$. This work, which came from the authors' own experience in conducting software projects, provided readers with a toolbox consisting of several steps, starting with traditional ones such as user stories and CRC cards. In a more specific study, Kamali et al. discussed several software testing methodologies that can be used in bioinformatics, such as simulators, testing in operational environment and cloud based software testing ${ }^{18}$. Artaza et al. proposed 10 metrics for life science software development, identified as the most relevant by a group of experts ${ }^{19}$. They include topics such as version control and software distribution or continuous integration. In a similar approach, Wilson et al. ${ }^{20,21}$ described a set of "good enough" principles that should be followed to better organize scientific computing projects, starting at the data gathering phase and continuing up to the writing of the manuscript. 
This paper leverages on the experience of the MedBioinformatics project, which primary aim is to develop integrative bioinformatics tools and software applications useful and autonomously usable by translational scientists and clinical practitioners. We present a set of recommendations for biomedical software development, with an explanation of how they can be implemented and what kind of open-source tools can be used for each specific topic. These recommendations can be adopted in any kind of software development, from user-interface applications up to scripts developed to support biology and clinical research, which are very often ignored from the software development point of view.

\section{Why should we care about software development recommendations?}

Many research organizations and teams can create biomedical software, but far too often, they are not sufficiently developed to be used by most clinical researchers and practitioners, because they are incomplete, lack user-friendly interfaces and software maintenance is not guaranteed after project completion. So, the main question we asked ourselves was how to ensure that the biomedical software development process in research institutes remained reliable and repeatable without them having to undertake major organizational changes.

Developing high quality biomedical software that accomplishes end-users' expectations implies following a minimal set of software engineering guidelines. We propose the following:

- Team and project management

- Tracking the development process

- Software integration and interoperability
- Test-Driven Development (TDD) and continuous integration (CI)

- Documentation

- Software distribution

- Licensing

Figure 1 presents a software development process that is following this general set of key steps. The first step, team and project management allows team members to keep track of group tasks and schedules, and be involved in development decisions. This encourages involvement of other users besides developers, who can point out missing features, give feedback and report bugs, helping communication between the whole team. Tracking the software development process consists of a combination of technologies and practices mostly used for source code management, but applicable to other collaborative tasks such as writing papers, product documentation, web site content, internal guidelines, and many more. Next, we have a cyclical pipeline between software integration and interoperability, which starts with the software specification phase and proceeds to the distribution phase, consisting of development, validation and deployment stages. The licensing of the software is one step that should be defined as early as possible, because during the development process it is often needed to include third-party dependency libraries, and the licenses should be compatible.

This test-driven development process can be used throughout the entire workflow, so that each unit is tested and the components' integration is validated. Moreover, the documentation of each

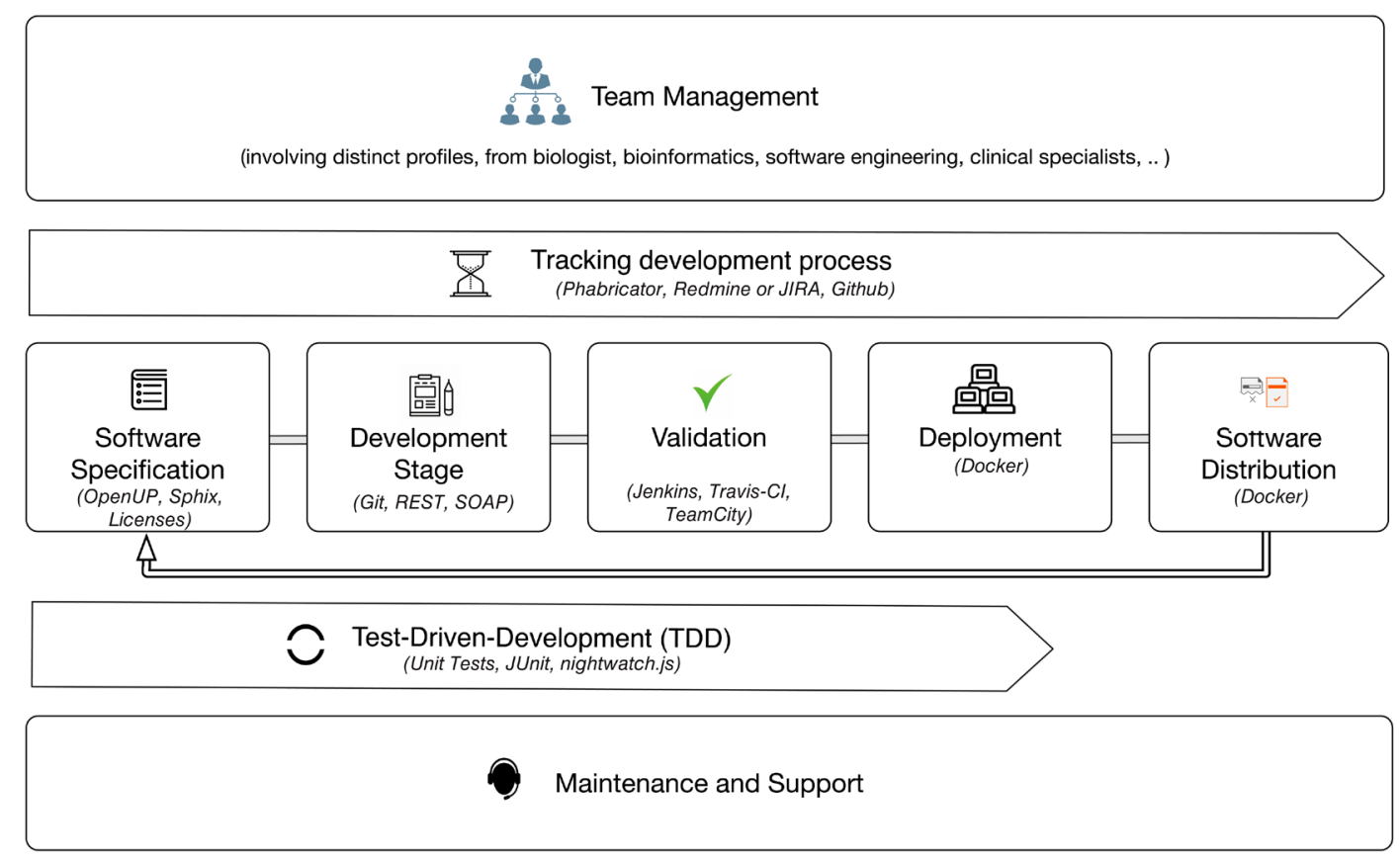

Figure 1. Software development process: including the several stages of the process. 
software module is important, and should be updated during all development phases. Finally, after the software application is distributed, appropriate maintenance and support is needed to assure end-users can rely on someone to handle their requests and help solve any problems.

To help the reader navigate through each of the following guidelines, we have divided each one into three sub-sections:

1) A summary that describes what it is intended for

2) The process description that explains what benefits it provides

3) Examples of tools and services that help to implement the guideline.

\section{Team and project management}

\section{Summary:}

Team and/or project management tools are essential for many organizations, to help in planning and organizing teams, tasks, and schedules. Implementing them during software development allows teams to stay synchronized about task scheduling and milestones, and helps track individual and general progress, identifying difficulties early on so that the necessary adjustments can be made. There are various software applications available that manage the development process; they typically include a variety of features for planning, scheduling, controlling costs, managing budgets, allocating resources, collaborating, and making decisions.

\section{Process description:}

Tracking and organizing the development process typically involves the following main features:

- Task management - To prioritize what functionality is developed over the different phases of project. It is often provided as a graphical user interface tool that uses the drag and drop functionality to facilitate project management, such as Kanban boards - a method to visualize and manage the workflow, where one can move the tasks between different phases;

- Code reviewing - This important practice is often used to support teams of multiple developers, despite also being very useful to track the progress of a single developer. These tools allow the code to be audited by providing differential views of code changes, normally web-based interfaces where reviewers/auditors inspect the code independently, from their own machines, as opposed to synchronous review sessions where authors and reviewers meet to discuss changes;

- Source code repositories - A source code repository is a web hosting facility to store and manage source code and which normally supports version control;

- Bug tracking - Keeps track of all defects and problems with the source code, using a predefined nomenclature to describe each issue.

The process typically also includes document repositories, wikis, discussion forums, time-tracking, Gantt mapping, file storage, calendars and versioning control.

The principles behind team and project management tools have been implemented in several software development methodologies, such as Lean and Agile, and are important aspects of Scrum methodology, Kanban and extreme programming (XP) ${ }^{22}$. Here, team management relies on several types of meetings, such as sprint planning meetings, daily Scrum meetings, sprint review meetings, sprint retrospective meetings and backlog refinement meetings. The Scrum Master is responsible for planning what will be discussed, namely what has been performed in the last sprint and what are intended to be done for the next sprint - a sprint is a specific period in which a set of tasks need to be accomplished. Developers also need to be prepared to analyse their development process, and negotiate future plans and potential deadlines. While Agile methodologies can lead to too many meetings, it is highly recommended to meet periodically to coordinate the development process.

\section{Examples:}

Depending on the type of financial resources available, free or open source management applications can be adopted, installed locally or used as a service in the cloud. Some examples of management applications are: Phabricator, Redmine or JIRA, Github and Bitbucket.

\section{Tracking the development process}

Summary:

A source control management system (SCM) provides coordination and management services between members of a software development team. It could be implemented in many different ways, and the most basic level, it could be a shared folder, and only the newest versions of files are available for use. In software programming, when there are several team members, the concept of branches is very important. Quite often, projects are only supported by a single researcher, but this is also very important for these small projects. To correctly support the concept of branch, more complex software is required.

\section{Process description:}

The more recent versions of SCMs allows developers to work simultaneously on the same file, merge changes with other developers' changes and track and audit changes that were pull requested. Nowadays, SCMs often include components to assist the code revision and also to manage software process milestones and roadmaps.

There are several strategies to develop with Git, and in this section a short summary of Git Flow is presented - a well-known branch model developed by Vincent Driessen ${ }^{1}$. The development process includes two branches: master and dev. Master will be the most stable branch. Only bug fixing can be merged in the master branch and the bug fix branches should always be pull requested to master. The Dev branch contains new features, and more unstable branches may be pull requested to this branch. This is where the developers are creating new features for the planned next releases of the software. Figure 2 shows an example of the bug fixing flux that occurs while a new branch is created from the master.

The process usually starts with an issue being reported, and after a decision has been made, it is assigned to a developer. Before going

${ }^{1}$ http://nvie.com/posts/a-successful-git-branching-model/ 


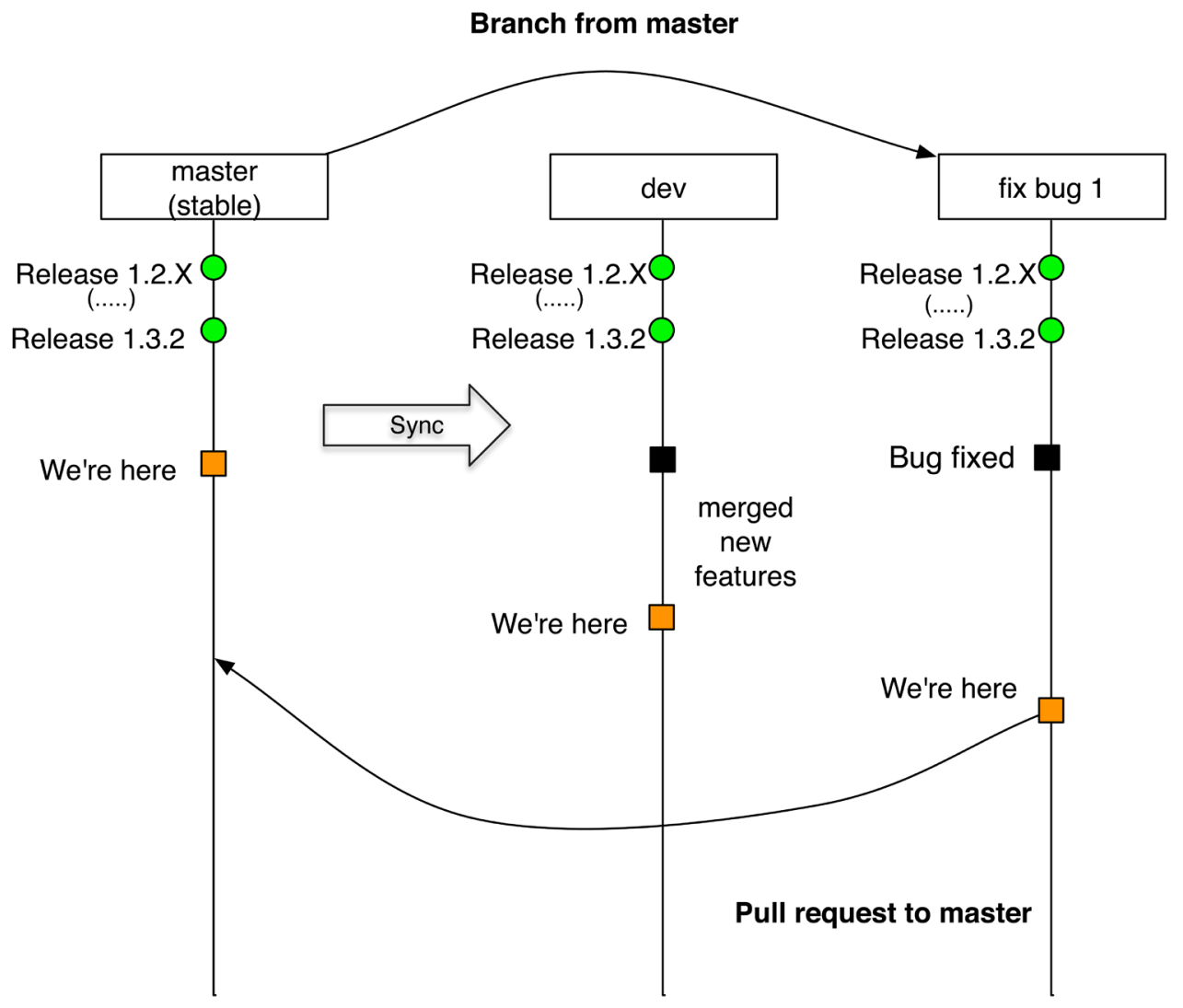

Figure 2. Example of a strategy for SCM workflow based on Git. It is an example of a bug fix branch from master branch and created a pull request with the changes against master branch.

to production, it needs to pass internal tests overseen by an internal testing team. If the bug has been fixed according to requests, the case is closed, or a report is sent back to the developer with a new set of issues.

New features are developed according to users' feedback. It is a complex task that often involves re-engineering the applications. This process may break some other features already in place. Thus, the new features are implemented in a development branch, passing through several analyses, tests and user feedback stages. Finally, release management is also performed within the SCM. Generally, it uses an incremental numbering schema to tag each version. In this way, it is always possible to track older versions and roll back to a previous version, which is mainly required to compare the behaviour of different versions.

The following best practices should be applied to software version control:

- Before committing, check for possible changes in the repository
- When committing a change to the repository, make sure the change reflects a single purpose (E.g. Fixing a bug, adding a new feature);

- If possible, try to create change sets linked to the issue tracker. Use the issue ID in the commit message;

- After merging, run the unit tests to ensure that the merge was successful;

- After creating a tag, do not commit to it any more. Visualize the tag as read-only. If it is necessary to resolve an issue in that specific version, create a branch from that tag and commit the changes to it;

- Try not to merge a large number of changes between the trunk and the branches. Use atomic commits;

- Make at least one commit per day with all the day's work.

\section{Examples:}

Several version control systems (VCS) can manage code development, such as Git or Mercurial. Github or Bitbucket are some examples of ready-to-use SCM. 


\section{Software integration and interoperability}

\section{Summary:}

Software integration and interoperability with external systems is a very important requirement in the biomedical domain, due to the reusability of existing repositories, services, algorithms, components and even applications. Designing an application programming interface (API) is crucial in distributed system development, so that the final solution can interconnect and interoperate with other systems.

\section{Process description:}

A programming interface exposes part of a system behaviour, and it is sometimes difficult to implement when different platforms and programming languages are required. Since creating a new interface for each specific service could be tiresome and errorprone, it is often preferred to take a generic interface and express application-specific semantics to them. This is often a trade-off between performance, extensibility and stability of the API. To collaborate with specifying new semantics and the development of systems complying with such interfaces, Interface Description Languages (IDLs) emerged as formal definition languages for describing software interfaces, often coupled with facilities for documenting the API and generating consumer and provider code stubs for multiple platforms or programming languages.

Two of the most used types of API are $\mathrm{SOAP}^{2}$ and REST ${ }^{23}$ :

- The Simple Object Access Protocol (SOAP) is an Internet protocol for messaging and remote procedure calls, using Extended Markup Language (XML) as the base message format and usually (although not necessarily) HTTP as the transport protocol. Web Service Definition Language (WSDL) is a commonly used IDL for describing a web service using SOAP. This protocol was very popular in its conception but is nowadays becoming replaced by other solutions such as REST.

- Representational State Transfer (REST) is an architectural style that defines an interface as a means of accessing and manipulating well-identified resources, using HTTP as the transport protocol and a set of methods for reading and writing resource state. REST is praised for its simplicity, performance, scalability and reliability. In the scope of web applications, client modules for consuming RESTful services can be easily implemented without the need for complex external libraries.

Defining an API is very important for software reusability, to ensure that developers allow their services to be integrated in thirdparty applications. In the biomedical domain, besides the existence of REST web services, use of well-defined standards and vocabulary is also crucial.

\section{Examples:}

Web service facilities are generally included in software development toolkits and for several programming languages.

${ }^{2}$ https://www.w3.org/TR/soap
Test-Driven Development (TDD) and Continuous Integration (Cl)

\section{Summary:}

The Test-Driven Development methodology is a software development technique based on short cycles. The basic idea is that the developer creates a set of test cases and writes those test cases to ensure a specific use case. A set of assertions should be established in each test, helping developers to better identify the requirements for each component of the software. As a complement to TDD, Continuous Integration (CI) is a development practice that automates the build, allowing teams to detect early problems.

\section{Process description:}

In a software development journey, there are often several strategies to bug fixing, and changing the behaviour of modules may introduce problems in other parts of the software. There are three strategies that could be used to tackle the issue:

- Unit and integration tests - Tests written by the programmer to verify if that particular part of the code respects the contract, i.e. what the input and the output is. Integration tests are often built to verify if the different pieces of system work together.

- Continuous integration - A practice that incorporates automatic builds, and allows the teams to detect problems earlier.

- TDD - The practice of writing the tests before writing the code.

TDD can be applied not only with unit tests but also with interfaces. To develop unit tests for the core of the application, it depends on the programming language. The methodology is simple, but application might be more complex. There is always a trade-off between the overhead it introduces and its benefit, so it can be adapted according to specific needs, e.g. validation of critical processes, as is common in the biomedical domain. TDD allows writing of code that automatically verifies if the produced output of an algorithm is as expected ${ }^{24}$. These tests can be used at any time, allowing to better deal with future changes in code, and saving time in future updates.

TDD and CI make the development process smoother, more predictable and less risky, even in advanced stages of the software lifecycle. Additionally, bugs can be traced and solved sooner, as they are continuously introduced into the project code. CI proposes the following set of development guidelines:

- Do not check in on a broken build;

- Always run all commit tests locally before committing;

- Commit your changes frequently (at least once a day);

- Never go home with changes to commit;

- Never go home on a broken build;

- Always be prepared to revert to the previous revision;

- Take responsibility for all breakages that result from your changes;

- Fix broken builds immediately. 


\section{Examples:}

An example of a tools that can be used for TDD is JUnit for java. To test web interfaces, there is the nightwatch.js tool, amongst others. For CI there are tools such as Jenkins, Travis-CI or TeamCity.

\section{Documentation}

\section{Summary:}

Documentation is one of the most important aspects of long-term software development. Building comprehensive documentation is very important for software reusability and maintenance, helping to mitigate the arrival/departure of team members Nevertheless, biomedical research software is often born based on experiments and scripts, and researchers are often not willing to document all processes and source code.

\section{Process description:}

High-level requirements intend to depict what the system "will be", rather than what it "will do". The emphasis is therefore on non-functional or business requirements. As the project evolves, these requirements will be progressively more detailed, and eventually converge with low-level requirements. Use case analysis is important for any development project, and it is a task usually shared with end-users. It is important to choose a simple and comprehensive use case template, and sometimes a first iteration with a key user can help refine it before distributing the template among all users.

Other technical documentation needs are mostly related to the project set-up, where a wiki system can be used for storing dispersed information in a controlled environment where everyone is able to edit/comment. This repository can include use cases, architecture/database diagrams, user interface mock-ups, and any project-related documents.

Last but not least, inline source code documentation is very important to define and explain the different parts of the source code, making it easier for the programmers when they need to add extra features or fix bugs. The code must be self-explanatory using an adequate name convention. The inline source code documentation must describe what the code does, how it works, and, when applicable, how it can be integrated with other pieces of code. Nowadays specific and automatic API generation documentation tools allow creation of easy to read documentation based on inline source code documentation.

\section{Examples:}

For general documentation, Markdown or Sphinx ${ }^{3}$ (also used for Python) can be used. For Java language, there is Javadoc, while other languages have their own documentation strategy that can be followed. For software specification and requirement analysis there are several templates in OpenUP ${ }^{4}$ (Open Unified Process) from Eclipse Foundation.

\section{Software distribution}

\section{Summary:}

Web-based solutions can be deployed in web servers, which makes life a lot easier for the application's end-users who do not need to deal with local installation. It is essential to handle updates smoothly without disrupting the quality of service provided.

\section{Process description:}

The deployment stage of each new release must not be performed in the production environment. It should follow three release management steps: development, testing and production (Figure 3). These distinct stages have similar conditions and they are deployed over different servers. Also, the production data is replicated in these environments to guarantee that the deployment

${ }^{3}$ http://www.sphinx-doc.org/

${ }^{4} \mathrm{http}: / /$ epf.eclipse.org/wikis/openup/

\section{Development Team and testers}

\section{Information Reports}

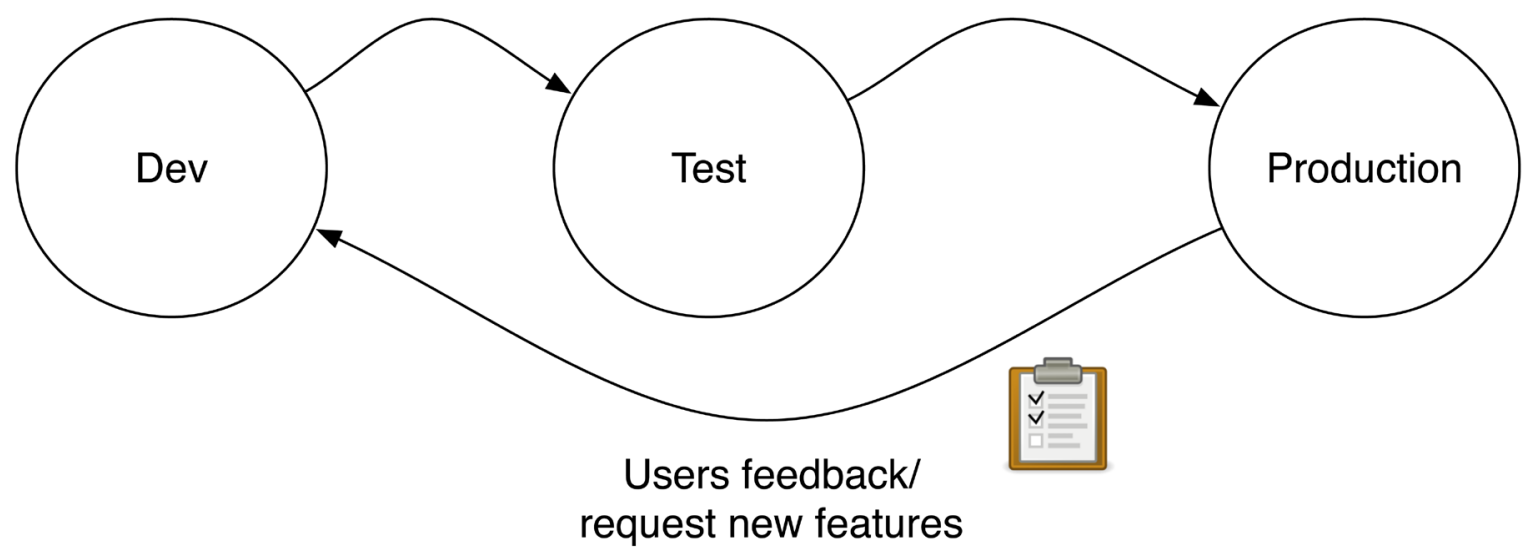

Figure 3. The deployment of each new release should follow three release management steps: development, testing and production. 
will be safely performed. Software engineers will often perform the development deployment and test the new features in this environment. When this milestone is reached, the release is performed and updated in the test stage. This version will be passed to a group responsible for testing, gathering feedback and feature enhancement. Once it has passed this stage, the final release will go into production to be used by the end-users.

\section{Examples:}

This is an organizational guideline, so no special tools are needed. Nevertheless, there are auxiliary tools that help the deployment and distribution process, mainly when the applications require complex setup tools. For example, it is possible to use software containers like Docker ${ }^{5}$ to distribute complex software and help deploy it, ensuring the whole community can run the software ${ }^{25-27}$.

\section{Licensing}

\section{Summary:}

Licensing and copyright attribution is a subject that should be addressed from the very beginning of the project. The goal is to clarify the terms that will regulate future use of the software - e.g. commercial, free use, open source. Open source software is currently a trend, even in bigger companies, as a way to credit the authors and promote work dissemination and collaborative development. Several kinds of licenses are available to regulate these relationships, although an individual disclaimer can be written. A commonly used license is the Free and Open Source Software (FOSS) license, which allows the product to be modified and redistributed without having to pay the original author.

\section{Process description:}

The license should be stated clearly on the project's front page and in the root of the source code. The full license text can be included here in a file called COPYING or LICENSE, following the standard format.

The copyrights should be assigned together with the license. The common nomenclature adds the year and the organization owning the copyright: Copyright $(C)<$ year $><$ name of organization $>$. The year specification may be a range, such as 2014-2016, to restrict the copyright to a period of time ${ }^{28}$. This line should be included in the headers of all source code files, together with a short license.

\section{Examples:}

There are different types of open source licenses, that come with different conditions and restrictions. We will list the most commonly used open source licenses:

- BSD License - It is the most permissive FOSS license. Users that re-use the code can do whatever they want, except in the case of redistributing source or binary, where they must always retain the copyright notice.

- Apache Public License 2.0 - This license is very permissive. It allows the licensed source code to be used in opensource and also in closed-source software.

\footnotetext{
${ }^{5}$ https://www.docker.com/
}

- GNU GPL - This license is restrictive. The users of the licensed system are free to use the licensed system without any usage restrictions; analyze it and use the results of the analysis (the source code must be provided and cannot be hidden); redistribute unchanged copies of the licensed system, and also modify and redistribute modified copies of the licensed system

- GNU LGPL - It is trade-off between the restricted GNU GPL and the permissive BSD. LGPL assumes that a library licensed under LGPL can be used in a non-GPL application. All the changes applied to the LGPL library must remain under LGPL. It assumes that all copyrights reversed on source code files, and not on the whole program.

\section{Conclusion and future directions}

In the biomedical domain, many new code scripts, algorithms, tools and services are currently being developed on a worldwide scale. However, the reuse of some of these software solutions outside the research lab is being hindered by them not following consolidated software developing methodologies. Early adoption of these methodologies is important in the development of biomedical tools so that they can reach a greater number of users; not only researchers but also healthcare professionals. During the development and distribution processes it is very important to involve end-users, to collect as much feedback as possible and create effective solutions during the development process.

We described a set of recommendations targeted at biomedical software developers aimed at achieving a good balance between fast prototyping, and robustness and long term maintenance. It is important to keep in mind that these recommendations are quite general and may not fit all cases, so adaptations may be required. We hope they can help biomedical researchers to reorganize their workflow, make their tools more visible, allow reproducibility of their research, and most importantly, that the outcome of that research can be more easily translated into daily clinical practice.

\section{Author contributions}

All authors participated in the discussions to achieve the software development recommendations. We believe all authors contributed equally to this work. All authors contributed to the writing and reviewing of this article. All authors read and approved the submitted manuscript.

\section{Competing interests}

No competing interests were disclosed.

\section{Grant information}

This work has partially received funding from the European Union's Horizon 2020 Research and Innovation programme for 2014-2020 under Grant Agreement n. 634143 (MedBioinformatics) and from the EU/EFPIA Innovative Medicines Initiative Joint Undertaking (EMIF grant $n^{\circ} 115372$ ).

The funders had no role in study design, data collection and analysis, decision to publish, or preparation of the manuscript. 
1. Sarkar IN: Biomedical informatics and translational medicine. $J$ Trans/ Med. 2010; 8: 22.

PubMed Abstract | Publisher Full Text | Free Full Text

2. Han D, Wang S, Jiang C, et al:: Trends in biomedical informatics: automated topic analysis of JAMIA articles. J Am Med Inform Assoc. 2015; 22(6): 1153-1163.

PubMed Abstract | Publisher Full Text | Free Full Text

3. Maojo V, Garcia-Remesal M, Bielza C, et al:: Biomedical informatics publications: a global perspective. Part II: Journals. Methods Inf Med. 2012; 51(2): 131-137.

PubMed Abstract | Publisher Full Tex

4. Gehlenborg N, O'Donoghue SI, Baliga NS, et al:: Visualization of omics data for systems biology. Nat Methods. 2010; 7(3 Suppl): S56-S68. PubMed Abstract | Publisher Full Text

5. He B, Baird R, Butera R, et al:: Grand challenges in interfacing engineering with life sciences and medicine. IEEE Trans Biomed Eng. 2013; 60(3): 589-598. PubMed Abstract | Publisher Full Text

6. Kulikowski CA, Shortliffe EH, Currie LM, et al.: AMIA Board white paper: definition of biomedical informatics and specification of core competencies for graduate education in the discipline. J Am Med Inform Assoc. 2012; 19(6): 931-938. PubMed Abstract | Publisher Full Text | Free Full Text

7. Umarji M, Seaman C, Koru AG, et al.: Software engineering education for bioinformatics. In 2009 22nd Conference on Software Engineering Education and Training. IEEE. 2009; 216-223. Publisher Full Text

8. Salman I, Misirli AT, Juristo N: Are students representatives of professionals in software engineering experiments? Proceedings of the 37th International Conference on, 2015. Publisher Full Text

9. Kane DW, Hohman MM, Cerami EG, et al:: Agile methods in biomedical software development: a multi-site experience report. BMC Bioinformatics. 2006; 7: 273. PubMed Abstract | Publisher Full Text | Free Full Text

10. Joppa LN, Mclnerny G, Harper R, et al:: Computational science. Troubling trends in scientific software use. Science. 2013; 340(6134): 814-815. PubMed Abstract | Publisher Full Text

11. Gymrek M, Farjoun Y: Recommendations for open data science. Gigascience. 2016; 5(1): 22.

PubMed Abstract | Publisher Full Text | Free Full Text

12. Pavelin $\mathrm{K}$, Cham JA, de Matos $\mathrm{P}$, et al:: Bioinformatics meets user-centred design: a perspective. PLoS Comput Biol. 2012; 8(7): e1002554. PubMed Abstract | Publisher Full Text | Free Full Text

13. Gentleman RC, Carey VJ, Bates DM, et al:: Bioconductor: open software development for computational biology and bioinformatics. Genome Biol. 2004; 5(10): R80.

PubMed Abstract | Publisher Full Text | Free Full Text

14. Nardi BA: A small matter of programming: perspectives on end user computing. MIT press, 1993.

Reference Source

15. Ko AJ, Abraham R, Beckwith $L$, et al.: The state of the art in end-user software engineering. ACM Computing Surveys (CSUR). 2011; 43(3): 1-44, 21. Publisher Full Text

16. Hastings J, Haug K, Steinbeck C: Ten recommendations for software engineering in research. GigaScience. 2014; 3(1): 31. PubMed Abstract | Publisher Full Text | Free Full Text

17. Prlić A, Procter JB: Ten simple rules for the open development of scientific software. PLoS Comput Biol. 2012; 8(12): e1002802. PubMed Abstract | Publisher Full Text | Free Full Text

18. Kamali $\mathrm{AH}$, Giannoulatou $\mathrm{E}$, Chen $\mathrm{TY}$, et al:: How to test bioinformatics software? Biophys Rev. 2015; 7(3): 343-352. PubMed Abstract | Publisher Full Text | Free Full Text

19. Artaza $\mathrm{H}$, Hong NC, Corpas M, et al.: Top $\mathbf{1 0}$ metrics for life science software good practices [version 1; referees: 2 approved]. F1000Res. 2016; 5 pii: ELIXIR-2000.

PubMed Abstract | Publisher Full Text | Free Full Text

20. Wilson G, Bryan J, Cranston K, et al:: Good Enough Practices in Scientific Computing. arXiv:1609.00037. 2016 Reference Source

21. Wilson G, Aruliah DA, Brown CT, et al:: Best practices for scientific computing PLOS Biol. 2014; 12(1): e1001745.

PubMed Abstract | Publisher Full Text | Free Full Text

22. Schwaber K: Scrum development process. In Business Object Design and Implementation. (eds. Sutherland, J., Casanave, C., Miller, J., Patel, P. \& Hollowell, G.) (Springer London). 1997; 117-134.

Publisher Full Text

23. Fielding RT, Taylor RN: Architectural styles and the design of network-based software architectures. 2000 Reference Source

24. Beck K: Test-driven development: by example. 2003. Reference Source

25. Belmann P, Dröge J, Bremges A, et al.: Bioboxes: standardised containers for interchangeable bioinformatics software. GigaScience. 2015; 4(1): 47. PubMed Abstract | Publisher Full Text | Free Full Text

26. Boettiger C: An introduction to Docker for reproducible research. ACM SIGOPS Operating Systems Review. 2015; 49(1): 71-79. Publisher Full Text

27. Di Tommaso P, Palumbo E, Chatzou M, et al:: The impact of Docker containers on the performance of genomic pipelines. PeerJ. 2015; 3: e1273. PubMed Abstract | Publisher Full Text | Free Full Text

28. Fogel K: Producing open source software: How to run a successful free software project. 2005

Reference Source 


\section{Open Peer Review}

\section{Current Peer Review Status:}

\section{Version 1}

Reviewer Report 05 April 2017

https://doi.org/10.5256/f1000research.11591.r21000

(C) 2017 Maojo V. This is an open access peer review report distributed under the terms of the Creative Commons Attribution License, which permits unrestricted use, distribution, and reproduction in any medium, provided the original work is properly cited.

\section{Victor Maojo \\ ${ }^{1}$ Biomedical Informatics Group, Artificial Intelligence Department, Universidad Politecnica de Madrid, Madrid, Spain \\ 2 Biomedical Informatics Group, Artificial Intelligence Department, Universidad Politecnica de Madrid, Madrid, Spain}

This is a timely report, given the proliferation of all types of biomedical informatics applications (from medical apps to laboratory or even complex clinical ones) delivered by software developers that do not follow even simple criteria of solid software engineering. In fact, many of these applications are built to carry out quite simple computational tasks (even, many times, quite successfully) but without a sound rigorous computing basis, and then are prone to multiple subtle errors or they lack standardized approaches and interoperability capacities. Besides the interest of the topic, the paper is well written, with a solid analysis of the topic, useful recommendations and a selected reference section, which can be very helpful to a broad range of readers, from public health informaticians to bioinformaticians. Below are some comments.

1. Although the authors are usually careful with this issue, readers outside the field may have some problems to understand the differences between medical informatics, bioinformatics, computational biologists and biomedical informatics. Sometimes the words are used in an interchangeable way in the paper, but this may lead to confusion. Some explanation might be necessary.

2. When the authors refer to "focus on decision support, NLP, information retrieval and EHRs" they mix techniques and a concrete system (EHR). They should explicit what technique they refer for EHRs.

3. The authors begin to address, apparently, biomedical informatics (thus, including public health and clinical topics) but they focus later in bioinformatics, which I believe it is the best target for the paper. Differences are usually significant between clinical (for instance, EHRs, with many big software companies dedicated to this field). Some example may be useful. 
4. Besides the provided hyperlink, some reference should be added for the MedBioinformatics project and a brief description.

5. The software engineering guidelines suggested are of interest, but some additional brief comparison and similarities/differences with established methodologies (besides what is presented for Test-driven) may provide additional insight.

6. As mentioned above, some brief, real example carried out by the authors may add some information of interest, pointing out actual problems and possible approaches. In fact, the paper is quite generic, but some specific case/application in the biomedical domain can be of interest.

7. Some differences may be pointed out when software developers work for an academic thesis or project, compared to a software company? Some comment may be of interest, too. In fact, many tools are quite simple, for a single task, and not intended for broader scenarios, where interoperability is necessary. Some recommendations could differentiate both cases.

8. For this reviewer, the paper may require some more explanation about design and prerequisites aspects, which are quite important, and some concrete example, but this is quite personal and the decision should be up to the authors.

Competing Interests: I have worked, around a decade ago, with one of the authors (Jose Luis Oliveira).

\section{I confirm that I have read this submission and believe that I have an appropriate level of expertise to confirm that it is of an acceptable scientific standard.}

Author Response 07 Jul 2017

\section{Luis Bastiao Silva}

Thank you for the positive assessment and helpful recommendations. We will answer point by point for your comments:

1) We agree, this discussion is important. We have included in this revision two new references where the explanation of the different fields is well addressed.

2) Done

3) Indeed, this is true and we tried to make it more clear along the article. Moreover, we included new references that discuss this issue in detail.

4) Thank you for highlighting this. A brief introduction to the project is now provided.

5/6) Indeed, we agree with this remark. However, since these recommendations result from the experience of several software projects, where many concrete use cases were explored, we also feel that detailing those could be out of scope of the article. 
7) Thank you for raising this, which is indeed a very important remark. We have now discussed this in more detail in the introduction section.

8) We agree with your remark. The design and prerequisites aspects are briefly addressed in the documentation process. We changed this section to highly better these issues.

Competing Interests: No competing interests were disclosed.

Reviewer Report 03 April 2017

https://doi.org/10.5256/f1000research.11591.r21001

(c) 2017 Rodrigues J. This is an open access peer review report distributed under the terms of the Creative Commons Attribution License, which permits unrestricted use, distribution, and reproduction in any medium, provided the original work is properly cited.

\section{João P. G. L. M. Rodrigues}

1 Department of Structural Biology, School of Medicine, Stanford University, Stanford, CA, USA

2 Department of Structural Biology, School of Medicine, Stanford University, Stanford, CA, USA

This review focuses on an important topic in modern bioinformatics: good practices for software development. As the authors note, there is a growing body of data derived from experimental studies that requires automated analysis. The analysis is often carried out using custom software, written by first-time or inexperienced programmers, and results in unsupported, sub-optimal, or duplicated code. As the authors also mention, several groups have tried to put forward a collection of tips and guidelines to help researchers in developing 'proper' software. This review offers a similar set of guidelines, targeted specifically at the field of biomedical informatics, and draws on the experience of the authors on building their own tools.

The suggestions cover seven topics, from management to in-depth software development tips, and do a very good job at explaining their importance and their take on what constitutes a good approach. The authors also give very good examples of software tools to help readers setup a development environment. These range from the usual 'use GitHub' to TravisCI, Sphinx, and Docker. One suggestion would be to integrate some of these tools in Figure 1, to give readers a visual cue where these tools fit in each topic/step. The authors also provide a very nice summarized view of the release process, namely licensing and distribution (e.g. using Docker), and the follow-up maintainance.

There is one less positive aspect of this review, which is anyway transversal to most such attempts at 'guidelines' for bioinformatics software development. As the authors note, most of these tools are created to solve one very specific problem, or process a very specific dataset. These are not amenable to test-driven development, or to continuous integration. More importantly, most of the authors of these tools/scripts are biologists, not programmers, which usually translates to a lack of interest in proper programming etiquette. Thus, I believe that it is important to show and teach 
such users very very simple programming rules, namely about how to make their code readable for others. For example, in the Python world, a simple recommendation to use 'flake8' to check for PEP8 coding standards and an editor (e.g. Atom, Sublime) that can do real-time code checks (typos, unused variables, indentation issues, etc). There is no need to suggest quasi-professional IDEs, as these will likely scare users away!

All in all, as a biologist doing bioinformatics and doing his best to follow proper software guidelines, I find reviews like this one very important to the field. They should probably feature in a 'starting package' to new PhD students in many labs. As an added suggestion, the authors could think of putting these guidelines in practice and follow up with a simple workshop/tutorial series, a la software carpentry, even if in webinar format.

Competing Interests: No competing interests were disclosed.

I confirm that I have read this submission and believe that I have an appropriate level of expertise to confirm that it is of an acceptable scientific standard.

Author Response 07 Jul 2017

\section{Luis Bastiao Silva}

Much obliged for your assessment and recommendations. We have redrawn Figure 1 following your suggestion. Regarding the second point, we recognised the importance of the subject and how recommendations vary according each developer/research profile and even programming language. For beginners or sporadic developers, most of the recommendation may not apply. However, this type of review creates the awareness of developing for the community, not just for ourselves. Finally, regarding last comment, indeed, guidelines for a new comers is good idea. We think this can be done at the institution level, since different methodologies may be used locally.

Competing Interests: No competing interests were disclosed. 
The benefits of publishing with F1000Research:

- Your article is published within days, with no editorial bias

- You can publish traditional articles, null/negative results, case reports, data notes and more

- The peer review process is transparent and collaborative

- Your article is indexed in PubMed after passing peer review

- Dedicated customer support at every stage

For pre-submission enquiries, contact research@f1000.com 